\title{
Research on the evaluation model and method of Smart city in small and medium sized cities
}

\author{
Yaqing QI \\ School of Beijing University of Technology, Beijing 100124, China; \\ qi_yaqing@126.com
}

Keywords: Smart city evaluation, Indicator weight, Analytic hierarchy process, Evaluation method

\begin{abstract}
This paper from the wisdom of urban construction and management to help managers of small and medium-sized cities that lack of expert resources to better grasp the wisdom of urban construction, study the advantages and shortcomings of analytic hierarchy process in the evaluation of Smart City, determine the index weight, at the same time improve the assessment method , and put forward a new model and method of Smart city evaluation, which was more suitable for the actual construction situation of small and medium-sized cities.
\end{abstract}

\section{Introduction}

At present, our country is actively promoting the construction of smart city, $100 \%$ sub provincial cities, $89 \%$ prefecture level cities and $47 \%$ county-level cities to carry out the wisdom of urban construction, And the upward trend is accelerating, But due to the fact that the city is different, All aspects of geographical, cultural and resource differences lead to some of the city's construction of a smart city appeared from the basis for urban development and the actual needs of the masses, and blindly launched projects resulted in waste of resources. These are seriously deviated from the wisdom of the city's original intention.

Although the relevant scientific research institutions, government departments are actively exploring, Such as Shanghai Pu dong New Area, published in the wisdom of Urban Construction Evaluation Index System 1.0 and wisdom City Construction Evaluation Index System 2.0, and Nanjing Information Center released report on smart city evaluation index system , China Intelligent Engineering Research Association released China smart city (town) development index evaluation system and Smart city SOA Standard Application Guide, Smart city technology reference model , Smart city evaluation model and basic evaluation index system and so on. These smart city national standards and evaluation system are added fast establishment.

But these smart city evaluation index system, the experts from the wisdom of the city itself, combined with the wisdom of the city development situation, reference first tier cities, the need for the construction of key cities. For the expert resources are relatively scarce in the small and medium cities, generally do not have the ability a suit oneself real smart city evaluation index system construction, and directly apply published wisdom city evaluation index system, the evaluation of urban results much to lose the scientificalness and correctness.

The based on this situation, combined with analytic hierarchy process (analysis, proposed a suitable for small and medium-sized cities in the wisdom of urban management evaluation scheme, provide tool and basis for the level of intelligence of the evaluation of urban construction, has an important significance to promote the healthy development of the wisdom of the city.

\section{Smart city evaluation model}

Smart city is a more advanced form of digital city, Application of Internet of things, cloud computing, big data and spatial geographic information integration technology, sensing, analysis and integration of the core system of the city's key information, So as to promote the transformation of government functions, to promote the innovation of social management, the infrastructure, public services, social management, ecological environment, industrial system more intelligent and optimized, so that people's lives better, the city is more harmonious. 
This paper selects wisdom City Construction Evaluation Index System 2.0 as the foundation of the smart city evaluation model. In the system, there are 6 indicators, two indicators, 18 indicators and three indicators, 37 indicators [1].

Because of the regional, economic, resource and other aspects of small and medium-sized cities, the convening of a certain influence of the forum, meeting and training to create a smart city atmosphere, at this stage is not easy to achieve, Influence on the development of smart city target limited, Three indicators two indicators so remove the wisdom of the city atmosphere and below, the wisdom of the city further evaluation model is more suitable for small and medium-sized city.

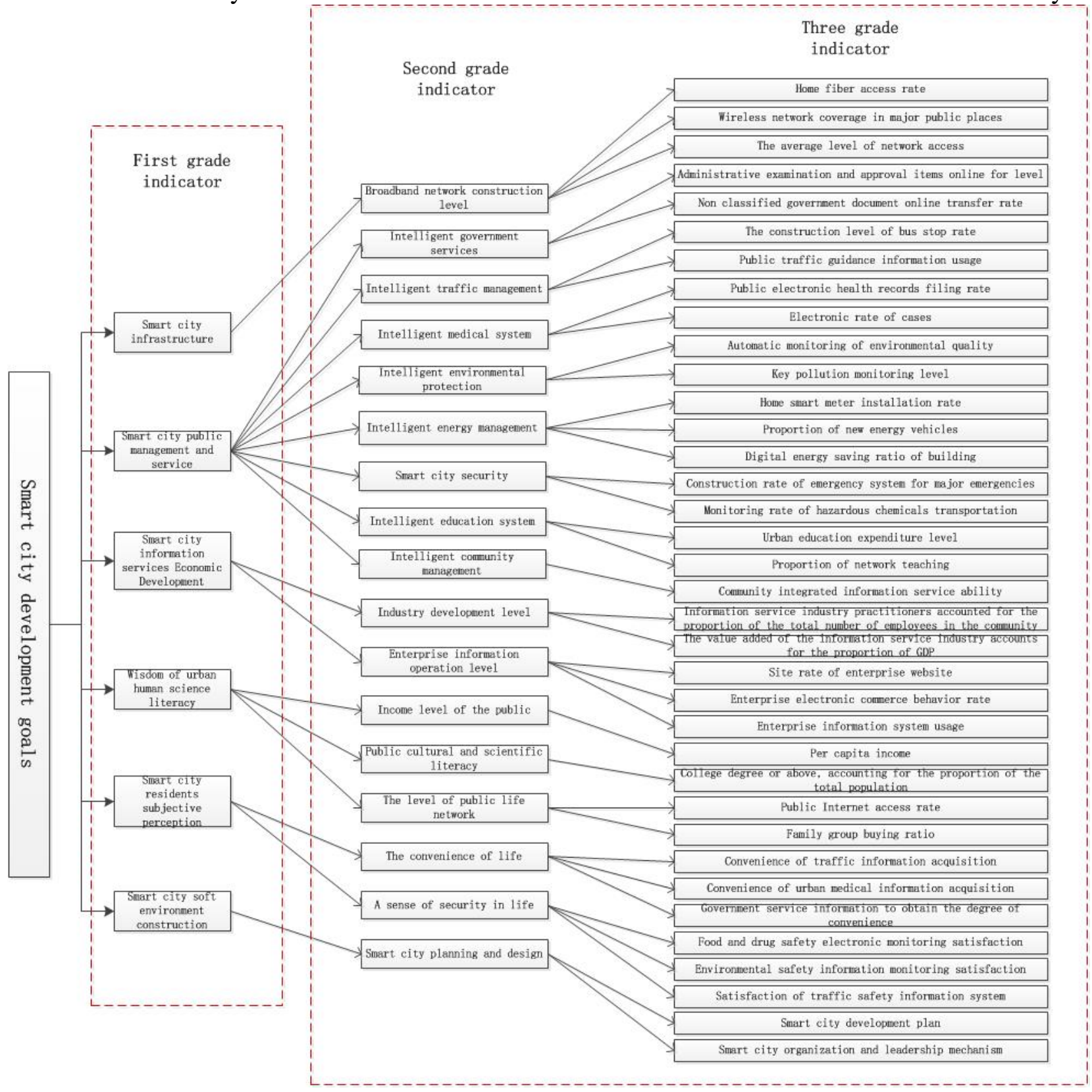

Fig.1 Smart city evaluation model

\section{Analytic hierarchy process to determine the indicator weight}

On the basis of the establishment of the basic model of intelligent city evaluation, Determine the impact of each index on the city's smart city development goal is the weight of the city, is the most important part of the wisdom of the city evaluation, the analytic hierarchy process is a very good method to determine the weights.

Analytic hierarchy process is a qualitative and quantitative analysis method of multi objective decision making, Make full use of the expert knowledge and subjective experience, and use 
mathematical quantization scale, we should try our best to eliminate the subjective elements, to determine the weights. Finally according to the consistency of the judgment matrix is satisfactory to determine the weights of rationality.

The traditional analytic hierarchy process is to establish the hierarchy model first, then construct the two comparison matrix, calculate the indicator weight, and finally carry on the consistency check [2].

There is a deficiency of the analytic hierarchy process when the number of indicators of each criterion is more than nine, will cause the annoyance of experts, leading experts to determine the chaos, quasi determined to reduce. Therefore, this paper uses grouping by using the analytic hierarchy process, the indicator as a group, second level indicators to level indicators as the standard to divide the group, three level indicators to second level indicators as the standard to divide the group.

Each group uses the analytic hierarchy process to get the relative indicator weight in the group. Sub module of the model:

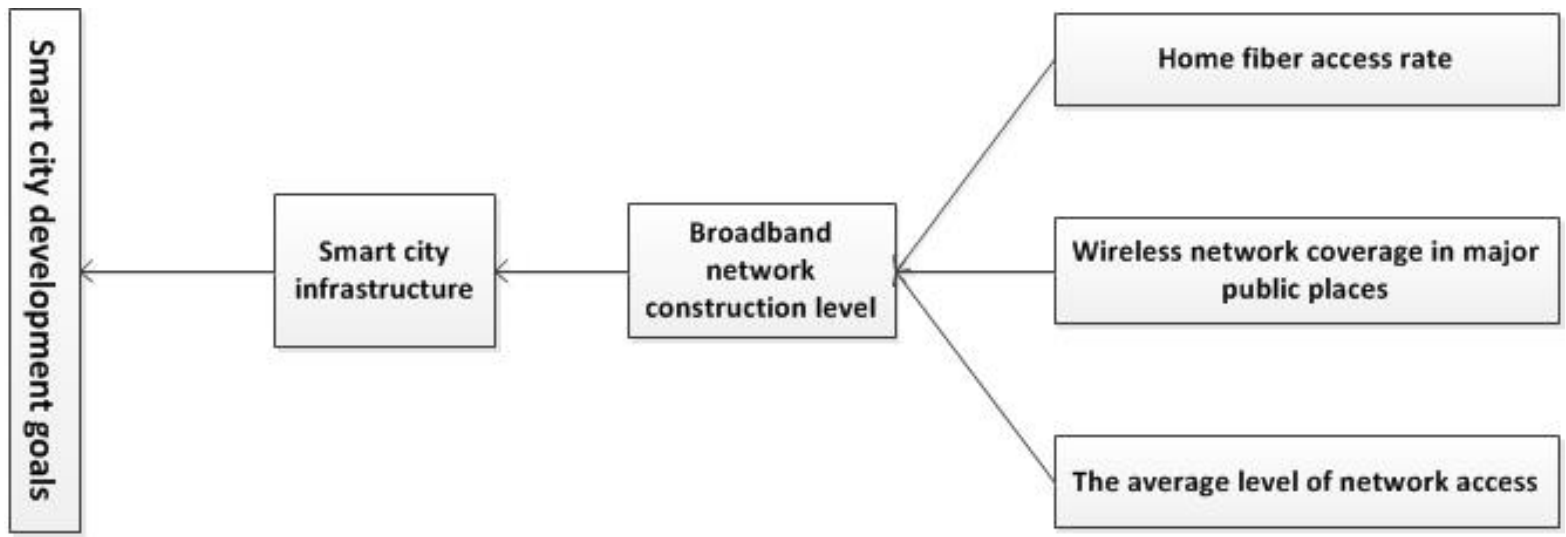

Fig.2 One sub module of smart city evaluation model

Suppose the first level indicator weight is as follows:

$$
\mathrm{I}_{1} \omega=\left\{\mathrm{I}_{1} \omega_{1}, \cdots, \mathrm{I}_{1} \omega_{6}\right\}
$$

Suppose the second level indicator weight is as follows:

$$
\mathrm{I}_{2} \omega=\left\{\mathrm{I}_{2} \omega_{1}, \cdots, \mathrm{I}_{2} \omega_{18}\right\}
$$

Suppose the three level indicator weight is as follows:

$$
\mathrm{I}_{3} \omega=\left\{\mathrm{I}_{3} \omega_{1}, \cdots, \mathrm{I}_{3} \omega_{37}\right\}
$$

In the sub module 1 , the relative weight of the first level indicator that is Smart city infrastructure is $I_{1} \omega_{1}$, the relative weight of the second level indicator that is broadband network construction level is $\mathrm{I}_{2} \omega_{1}$, the relative weight of the three level indicator that is home fiber access rate is $I_{3} \omega_{1}$, the relative weight of wireless network coverage in major public places is $I_{3} \omega_{2}$, the relative weight of the average level of network access is $I_{3} \omega_{3}$. Then the final weight of the three level indicators of family fiber access is as follows:

$$
\mathrm{W}_{1}=\mathrm{I}_{1} \omega_{1} \times \mathrm{I}_{2} \omega_{1} \times \mathrm{I}_{3} \omega_{1}
$$

the final weight of wireless network coverage in major public places is as follows:

$$
\mathrm{W}_{2}=\mathrm{I}_{1} \omega_{1} \times \mathrm{I}_{2} \omega_{1} \times \mathrm{I}_{3} \omega_{2}
$$

the final weight of the average level of network access is as follows:

$$
\mathrm{W}_{3}=\mathrm{I}_{1} \omega_{1} \times \mathrm{I}_{2} \omega_{1} \times \mathrm{I}_{3} \omega_{3}
$$

Other indicators in accordance with this method can be followed by three indicators of the contribution of the smart city development goals. That is, the final weight of the three indicators is as follows:

$$
\mathrm{W}=\left\{\mathrm{W}_{1}, \mathrm{~W}_{2}, \cdots, \mathrm{W}_{37}\right\}
$$

\section{Smart city assessment method}

According to the development level of domestic and international smart city, to develop the evaluation criteria of each evaluation indicator. 
The traditional grading standard is divided into five grades, excellent, good, secondary, qualified, and bad [3].

Tab 1 Evaluation of family fiber access rate

\begin{tabular}{cccc}
\hline Numble & Rank & Home optical fiber access & Score \\
\hline 1 & excellent & $>90 \%$ & $>85$ \\
2 & good & $>80 \%$ & $75-85$ \\
3 & secondary & $>70 \%$ & $60-75$ \\
4 & qualified & $>60 \%$ & $40-60$ \\
5 & bad & $<60 \%$ & $<40$ \\
\hline
\end{tabular}

And then the experts score the relevant indicators with the evaluation criteria. Finally, through the weighted calculation of each indicator, carry out summary, and then draw result which is the smart city's total score of the wisdom evaluation. This comprehensive score represents the level of smart city construction [3].

This paper, through the study of the model of the three 37 level indicators, most of the proportion data are available. Because small and medium-sized cities in the urban construction goals are similar, implementing a system of one hundred percent, the proportion data is multiplied by 100 , that is the score of the indicator. It eliminates the need for experts on each indicator to scoring link and saves the workload, improves the scoring efficiency. It can facilitate the management of small and medium-sized cities in the self-examination, better grasp the smart city construction and wisdom of the specific situation, and closer to the actual development of the city.

The indicator score formula for the proportion data is as follows:

$$
\text { Indicator score }=\text { Indicator ratio value } \times 100
$$

In addition, there are two indicators of the non-proportion data, respectively, smart city development planning and smart city organization and leadership mechanism. The score of these two indicators adopts the method of grade division.

Tab 2 Evaluation of smart city development plan

\begin{tabular}{cccc}
\hline Numble & Rank & Smart city development plan & Score \\
\hline 1 & good & develope and implement & $>80$ \\
2 & secondary & develope and not implement & $60-80$ \\
3 & bad & not implement & $<60$ \\
\hline
\end{tabular}

Tab 3 Evaluation of the leadership mechanism of smart city

\begin{tabular}{|c|c|c|c|}
\hline Numble & Rank & $\begin{array}{l}\text { Whether the local } \\
\text { government set up the } \\
\text { organization structure with } \\
\text { the function of overall } \\
\text { planning and coordination }\end{array}$ & Score \\
\hline 1 & good & Yes & $>60$ \\
\hline 2 & bad & No & $<60$ \\
\hline
\end{tabular}

Suppose $\mathrm{S}$ is the score of each indicator, then the scores of 37 indicators are as follows.

$$
\mathrm{S}=\left\{\mathrm{S}_{1}, \cdots, \mathrm{S}_{37}\right\}
$$

Finally, applying linear comprehensive evaluation method that is the weight of 37 indicators multiply by the score of relevant indicators, the add together. That is the score of smart city construction level.

\section{Summary}

This paper gives the smart city evaluation scheme, which is based on analytic hierarchy process to determine the indicator weight in the model. Analyze the limitation of analytic hierarchy process, and the use of the model is feasible. Evaluation method has changed the traditional classification, expert evaluation. Because small and medium-sized cities are in same level, and have 
characteristics of similar resources, scores are ratio, which can save the amount of work, and help the development of small and medium-sized cities to have a certain reference value.

\section{References}

[1]. Information on: http://www.pudong.gov.cn

[2]. Dianting Wu, Dongfang Li. The deficiency of analytic hierarchy process and the ways to improve it, J. Journal of Beijing Normal University. 2004, 40(2):264-267.

[3]. Chuntao Gao. Discussion on evaluation indicator and method of Intelligent City, J. Summary Research, 2015, (295):38-42. 